\title{
EL DESTELLO CROMÁTICO DE DELEUZE EN EL PERSPECTIVISMO DE VIVEIROS DE CASTRO
}

Julián García Labrador ${ }^{1}$

Resumen: El objeto del presente artículo es presentar la integración de la "intensidad" deleuziana en el corpus de la obra de Viveiros, especialmente en su Metafísicas caníbales. Para Viveiros de Castro la relación con la diferencia se construye desde la "predación". De esta manera, en las culturas amazónicas los enemigos son asimilados como afines cuando son depredados. Pero antes de que el proceso de metamorfosis devenga historia, el mito se eleva como la categoría central de comprensión. El mito es para Viveiros el régimen atemporal de diferencia ontológica intensiva. Todas las potencialidades de ser se reúnen en su tensión diferencial en el mito. Viveiros remite a Deleuze para comprender que el mito determina las condiciones intensivas del sistema. De esta manera, Viveiros hace de Deleuze el interlocutor privilegiado para comprender las dinámicas humanas de los pueblos amerindios desde la intensión y la extensión.

Palabras clave: Antropología; mito; intensidad; predación.

En Metafísicas caníbales el antropólogo brasileño Viveiros de Castro (2010a) indica que la colonización de la antropología contemporánea procede de la aplicación epistémica de las distinciones entre nosotros y ellos. La antropología que se construye desde esta incuestionada premisa arroja categorías tales como "no modernos", "no occidentales", e, incluso, "no humanos" sobre todos aquellos colectivos humanos representados como "diferencia". Por otra parte, en su trabajo de campo con los Areweté, Viveiros de Castro (1992) había descubierto que, en el pensamiento amerindio, lo diferente no ocupaba un espacio reservado para representaciones negativas, sino una potencial afirmación de transformación identitaria. Los Areweté, pertenecen al grupo Tupí Guaraní, quienes para los primeros misioneros, se convertían a la fe cristiana de manera inauténtica ya que adoptaban la apariencia y las prácticas de los conversos, sin realmente convertirse, ya que pronto volvían a sus prácticas rituales anteriores. Esto, que fue considerado como "inconsistencia" (Viveiros 2011), por jesuitas y capuchinos, revela más bien, que, desde el lado amerindio, la identidad no es un asunto cerrado. Es decir, para el pensamiento amerindio, lo diferente podría ser integrado o, mejor aún, convertirse en la propia identidad, ya que ni la identidad es una "entidad" cerrada, ni la diferencia es una declaración negativa. Así, cuando un chamán se transforma en jaguar está actualizando lo que ya estaba contenido en la declaración mítica primordial, donde no se daba la distinción entitativa entre los seres:

As is well known, a sizeable slice of Amazonian mythology deals with the causes and consequences of the species-specific embodiment of different agents, all of them conceived to have originally partaken of a generalized unstable condition in which human and nonhuman features are indiscernibly mixed (Viveiros de Castro, 2007, p. 17).

La experiencia amazónica fue también la inspiración del trabajo de Levi-Strauss, quien, para Viveiros de Castro, incorporó el pensamiento amerindio en su elaboración teórica. Según Viveiros de Castro el estructuralismo es, de alguna manera, la traducción conceptual de la configuración amerindia del mundo. Viveiros reconoce, así los "fundamentos amerindios del estructuralismo" (Taylor, 2004, p. 97) y se propone, de igual manera que Levi-Strauss, extender su elaboración teórica sobre la senda abierta por el pensamiento amerindio. No obstante, este

\footnotetext{
${ }^{1}$ Universidad Politécnica Salesiana - Quito - Ecuador. E-mail: 1abradorjg@gmail.com.
} 
esfuerzo de incorporación de lo diferente no podría ser llevado cabo sin la filosofía de DeleuzeGuttari. Este es el propósito del presente artículo.

\section{Una antropología predatoria}

El pensamiento de Deleuze-Guatari (1985) sirve de marco fundacional para la elaboración antropológica de Viveiros de Castro. Metafísicas caníbales plantea la construcción imaginaria de un Anti-Narciso, a imagen del Anti-Edipo. Si la antropología ha pretendido erigirse como la construcción teórica acerca del otro, lo ha hecho sobre la base de una metafísica occidental, la cual ha permitido no sólo la construcción del antropocentrismo, sino la asignación de propiedades a lo humano, distinguiendo entre humanos y no humanos. Según Goddard la metafísica occidental se nutre en Viveiros de Castro de tres supuestos: "es intra-específica (tiene lugar entre humanos), está fundada en una producción filiativa; repite la expresión prototípica del otro bajo la figura del amigo o del amado que hace de la philia el elemento del saber" (2016, p. 208). Es la metafísica occidental la que ha permitido decir quién tiene y quién no tiene mundo, dónde hay exceso, pobreza o carencia de ser. Por ello, para Viveiros de Castro es esta metafísica la fuente de todo colonialismo:

Nosotros, sólo nosotros los europeos somos los humanos completos, o si se prefiere, grandiosamente incompletos, los millonarios en mundos, los acumuladores de mundos, los 'configuradores de mundos'. La metafísica occidental es verdaderamente la fons et origo de todos los colonialismos" (Viveiros, 2010a, p. 20).

Con el fin de tantear una antropología en permanente estado de descolonización, Viveiros de Castro busca en Deleuze y Guattari la apertura post-metafísica que permita incorporar el pensamiento del otro amerindio sin subyugar ni clasificar su nivel ontológico ${ }^{2}$. Más que incorporar (lo cual supondría un punto de llegada), para Viveiros de Castro "no se trata de borrar los contornos, sino de plegarlos, de densificarlos, de irisarlos y de difractarlos" (2010a, p. 21), adoptando el "cromatismo generalizado" (Deleuze-Guattari, 1980, p. 123). Es así como el Anti-Narciso, borrador imaginario que de-construye la metafísica occidental, se convierte en "antropología cromática", irisación potencial de los mundos que habían sido negados por la metafísica occidental.

Sobre el fondo fundacional deleuziano, Viveiros escribe su antropología en diálogo con aquellos referentes que han puesto en cuestión la distinción entre lo mismo y lo diferente. De esta manera, esboza un lienzo impresionista donde sólo la mirada distanciada podría otorgar sentido al conjunto. Por un lado, con el cuestionamiento del mismo concepto de cultura y de la tarea del antropólogo, Roy Wagner llamó la atención sobre la semiótica de la invención, distanciándose de la metodología clásica del trabajo etnográfico. Al proponer su "reverse anthropology" (1981, p. 30), traducida por Viveiros como "retro-antropología" (2010a, p. 21), Wagner asume el proceso de invención cultural del Otro, densificando la diferencia en la producción antropológica. Para Viveiros de Castro, Roy Wagner va a ser un referente obligado, un tamiz para cernir la luz de Deleuze en la metodología antropológica. Si Viveiros de Castro rastrea las raíces amerindias del estructuralismo lo realiza aplicando metodológicamente la propuesta de Wagner. Por otra parte, de Bruno Latour Viveiros de Castro rescata el cuestionamiento de los contornos, el esfuerzo por borrar las fronteras. Así, la sospecha

\footnotetext{
${ }^{2}$ Es esta una de las diferencias fundamentales entre Descola y Viveiros de Castro, puesto que, mientras Descola sigue anclado en sistemas clasificatorios y tipológicos, Viveiros de Castro abre su perspectivismo a constante revisión e inestabilidad. Ver Latour, B. 2009. "Perspectivism: Type or Bomb?". Anthropology Today, guest editorial, v. 25, n. 2, p. 21-22.
} 
latouriana sobre la distinción entre humano y no-humano es ocasión para que Viveiros afiance conceptualmente el perspectivismo amazónico como un continuum entre especies. Además la impugnación de la distinción modernos y no-modernos diseñada por Bruno Latour (1993) como un dispositivo de sombra permanente sobre la metafísica occidental, es actualizada por Viveiros de Castro en su de-construcción de cualquier antropología taxonómica. Por último, el brillante ensayo de Marilyn Strathern (1988) sobre el don en melanesia le permite a Viveiros la asunción de una definición abierta y no tipológica de cultura. La cultura sería un "multidimensional dispositivo de comparación" (Viveiros, 2010a, p. 70) ya que consistiría en los modos en que las personas establecen "analogías entre los diferentes dominios de sus mundos" (Strathern 1992a, p. 47). Por eso, desde la "anticrítica melanesia de la razón occidental" (Viveiros 2010a, p. 21), Strathern es la autora más deleuziana de todos sus referentes. En efecto, la apertura conceptual de Strathern para ir más allá de lo "uno" es fundamental para que Viveiros afirme: "la antropóloga de Cambridge, que comparte con Deleuze-Guattari un conjunto de términos conceptualmente densos, como multiplicidad, perspectiva, di-vidual, fractalidad, es, en varios aspectos, el autor más 'molecularmente' deleuziano entre las tres citados" (2010a, p. 92)

Este panorama, enriquecido en sus pliegues hasta una densificación posiblemente sin término, contribuye al esbozo de antropología cromática que pretende llevar hasta sus últimas consecuencias el anuncio programático de Levi-Strauss:

nos agrade o nos inquiete, la filosofía ocupa de nuevo el centro del escenario. Ya no nuestra filosofía, de la que mi generación pidió a los pueblos exóticos ayuda para deshacerse; sino, por un asombroso retorno de las cosas, la de ellos (Lévi-Strauss, 2000, p. 720).

El pensamiento de los pueblos exóticos se convierte así en la senda de una nueva antropología, de una exploración amerindia donde el uno y la diferencia no encuentran su frontera sino en los contornos de la metafísica occidental. Viveiros de Castro ejerce así de anfitrión y de huésped, de antropólogo que recibe en su ejercicio el don de la diferencia amerindia y de invitado occidental llamado a desestabilizar y de-construir permanentemente el discurso unificado. Y es esta relación entre anfitrión y huésped la que, en el pensamiento amazónico de los Tupí-Guaraní, se descubre como canibalismo (Viveiros, 1992). La práctica caníbal implica una metafísica de predación (Andrade 1995), según la cual "hacerse otro" no consiste sino en devorar la diferencia. La intención de Viveiros de Castro "no es otra que profundizar en las prácticas amerindias del canibalismo como un proceso de incorporación del otro y de excorporación de uno mismo" (Bouhaben, 2017, p. 98). Es por ello que la antropología cromática de Viveiros deviene antropología caníbal cada vez que la Diferencia y el Uno se aprecian como polos de un continuum predatorio.

\section{Diferencia y representación}

La predación amazónica como camino para "hacerse otro" implica que los polos se asimilación impiden pensar la identidad como entidad terminada o cerrada. Uno siempre podrá convertirse en otra cosa por un acto de predación. Esto impide la consideración de la identidad en términos entitativos. Viveiros de Castro recurre, por ello, a la deconstrucción del sujeto de la antropología de Strathern y a la incorporación del concepto de "di-vidual" como alternativa al "individual" de la metafísica occidental. Si bien, como reconoce Aparecida Vilaça (2016), Strathern toma "dividuality" del trabajo de Marriot (1976), en la investigación amazónica se convierte en una noción clave del perspectivismo. 
En efecto, la antropología de Viveiros de Castro es comúnmente conocida como perspectivismo - término tomado del trabajo de Århem con los Makuna (1993) -, ya que las perspectivas son los polos del contiuum que hemos descrito. Ser Uno y sí mismo y ser la Diferencia, es sólo cuestión de perspectiva. Ser presa o predador dependerá de la perspectiva: "cuando pasa la lengua por la sangre de su presa abatida en la selva, el jaguar no ve ese líquido como sangre cruda, sino como cerveza hecha de mandioca fermentada" (Viveiros, 2013, p. 57). Se trata, empero de una perspectiva no representacional, sino constitutiva. "Es el sujeto quien pertenece a una perspectiva y no al revés" indica Viveiros (2013, p. 82), apuntando a la destrucción del sujeto representacional cartesiano. Continúa en esta crítica deconstructiva cuando añade:

"El punto de vista crea el sujeto" -ésta es una proposición perspectivista por excelencia, la que distingue el perspectivismo del relativismo o del construccionismo occidentales, que afirman, por el contrario, que "el punto de vista crea el objeto" (Ib, p. 82).

Entre presa y predador sólo media la perspectiva, de igual manera que las relaciones entre humanos y no-humanos se establecen por las perspectivas con las que unos y otros intercambian sus posiciones, impidiendo levantar el abismo que Viveiros de Castro, siguiendo a Latour, critica en la metafísica occidental. Precisamente por eso, Viveiros de Castro corrige la posición de Quine (no hay entidad sin identidad) afirmando que "If no entity without identity, then no multiplicity without perspective" (Viveiros, 2010b, p. 224). Nos encontramos así con la reivindicación más pura de la multiplicidad: el perspectivismo constitutivo. "Pero entonces, si la perspectiva es algo que constituye al sujeto, ésta sólo puede aparecer como tal a los ojos de otro. Porque un punto de vista es pura diferencia" (Viveiros, 2013, p. 82).

Considerar que la perspectiva es "pura diferencia" nos lleva de nuevo a Deleuze, donde confluye, efectivamente, la diferencia con la crítica al pensamiento representacional, al igual que ocurre en el paradigma amerindio.

Uno de los hitos que el pensamiento amerindio levanta contra la antropología clásica es el cuestionamiento del régimen de representación en que ésta se hallaba comprometida. Significar el mundo no se acompaña de sus representaciones. Por ello, el grafismo de los pueblos amerindios, calificado como "inconofóbico" por varios autores (Taylor 2010, Clastres 2004, Lagrou 2007), es sumamente rico en significaciones no representativas (García y Vinolo 2018). El grafismo amerindio presenta la Diferencia sin congelarla de manera representativa. Se trata más bien de sugerir perspectivas. Como bien advierte Viveiros de Castro, al rechazar la representación contemplativa, entramos en el juego de la interacción comunicativa:

A ese achatamiento ontológico viene a corresponder una epistemología simétrica (Latour, 1991). En rigor, asistimos a la disolución de la distinción entre epistemología (lenguaje) y ontología (mundo) y a la emergencia progresiva de una "ontología práctica" (Jensen 2004) en la que el conocer ya no es un modo de representación desconocido, sino de interactuar con él, es decir un modo de crear más que de contemplar; de reflexionar o de comunicar (Deleuze y Guattari, 1991) (Viveiros de Castro, 2010, p. 96)

¿Por qué Deleuze? El enfoque deleuziano nos remite precisamente al "estatuto ontológico de la diferencia" (Biset, 2011, p. 7). En lugar de considerar la diferencia como un momento de la dialéctica (propio de la corriente hegeliana), la diferencia es en Deleuze una diferencia afirmativa. Se trata de una distinción fundamental y justificación de la elección deleuziana de Viveiros de Castro. En lugar de elegir una diferencia al estilo de Hegel (como negatividad del proceso) o 
Derrida (1989) (entendiendo la dialéctica como una economía de sentido), la diferencia deleuziana permite pensar la diferencia sin un concepto. Viveiros de Castro se da cuenta de que al discurrir por la senda de Deleuze afirmando ontológicamente la diferencia se aleja de cualquier pretensión taxonómica de la antropología. En efecto, la diferencia de Deleuze repudia tanto la lógica identitaria hegeliana, como la finitud representacional aristotélica. El distanciamiento respecto a Hegel lo encontramos en el rechazo a la subordinación de la diferencia "pues la diferencia no implica lo negativo, y no admite ser llevada hasta la contradicción más que en la medida en que se continúe subordinándola a lo idéntico" (Deleuze, 2002, p. 15). El estatuto ontológico de la diferencia no puede ser para Deleuze una mera mediación de lo idéntico. La diferencia es "primera respecto de la negación" (Biset, 2011, p. 19). Esta primacía ontológica otorgada a la diferencia es también el motivo para rechazar la dinámica representacional. Según Deleuze la representación supondría el sometimiento de la diferencia al subordinarla al concepto:

El error de la filosofía de la diferencia, de Aristóteles a Hegel, pasando por Leibniz, fue tal vez haber confundido el concepto de la diferencia con una diferencia simplemente conceptual, contentándose con inscribir la diferencia en el concepto en general (Deleuze, 2002, p. 58)

Tanto Hegel como Aristóteles habrían sucumbido a esta tentación, la de anular ontológicamente la diferencia, bien con la representación finita de Aristóteles al subordinar la diferencia a la clasificación en géneros y especies, bien con la representación infinita de Hegel al mediatizar la diferencia como fundamento de la totalidad (Biset 2011).

Viveiros de Castro ve confirmada la sospecha anti-representacional en el trabajo etnográfico. Al igual que Deleuze (1996) fue la empiría el primer motivo para sospechar de la clasificación y la subsunción: la diferencia aparece en la misma experiencia y no tanto como producto cognitivo o clasificatorio. Tanto es así que Viveiros de Castro rechaza en un mismo plano dialéctica y teleología, dando al traste con las posibilidades subordinacionistas de la representación:

El problema, en último análisis, consiste en construir un concepto no contractualista y no dialéctico del intercambio: ni interés racional, ni síntesis a priori del regalo; ni teleología inconsciente, ni trabajo del significante; ni fitness inclusivo, ni deseo del deseo del Otro; ni contrato, ni conflicto, sino más bien un modo de devenir-otro. La alianza es el devenir-otro propio del parentesco. (Viveiros, 2010a, p. 241).

¿Qué queda, entonces? La mera afirmación de lo dado. Es este un punto de convergencia, mejor aún, de partida del enfoque post-estructural de Viveiros y de orientación fenomenológica de Ingold (2000). Viveiros de Castro sugiere esta similitud en una nota de inicio de Metafísicas caníbales (2010a, p. 22), aunque no avanza en las posibilidades que podrían abrirse. Esta fidelidad a lo dado implica, efectivamente, la renuncia al régimen representacional, como evidencia la crítica a la taxonomía que hemos presentado. No parece, entonces, acertado el reproche de Martins y Feronato cuando, denunciando que Viveiros no abandona el locus de enunciación de la antropología clásica, avanzan indicando que "recriminaba al Estado representativo sin criticar, en ningún momento, la lógica misma de la representación” (2017, p. 485). ¿No es suficiente la renuncia de Viveiros a la representación ontológica de la diferencia? Desde mi punto de vista la incursión en la filosofía de Deleuze no podría haberse hecho sin la renuncia a la dinámica de representación, cuya dimensión ontológica es independiente de cualquier funcionalidad política: "la política no tiene un vínculo privilegiado con la ontología, no existe una relación necesaria entre una y la otra" (Biset y Farrán, 2011, p. 7). Es decir, si 
como continúan los autores "la política es aporética, es decir, al mismo tiempo que está saturada por múltiples definiciones existe una falta que imposibilita esa saturación" (Ib, p. 7), no podríamos concluir que de la misma ontología de Viveiros podamos obtener una posición política saturada.

La fidelidad a lo dado que, en Viveiros (2013) se traduce como "perspectivismo" y en Ingold (2017) como "correspondencia", nos permite pensar la multiplicidad de las prácticas humanas como trazos de luz en el entorno, como un paisaje donde los contornos nunca fueron borrados, como un conjunto cromático al más puro estilo de Deleuze.

\section{Intensidad y virtualidad en el pensamiento amerindio}

Uno de los ejemplos más bellos del cromatismo deleuziano en Viveiros de Castro es la generación de la diferencia en términos de virtualidad mítica. Viveiros parte de la distinción deleuziana entre intensivo (virtual) y extensivo (actual) para desmontar las categorías clásicas de "filiación y alianza", categorías que han servido para definir los límites de lo humano. Partiendo del concepto de "rizoma" de Deleuze, Vivieros de Castro presenta una concepción rizomática del parentesco. Si para Strathern "las relaciones son parte integrante de las personas" (1992b, p. 101), Viveiros de Castro entiende que la teoría del parentesco hay que reformularla asumiendo la correción a Quine de la que hablábamos anteriormente. Una teoría del parentesco perpectivista no podría construirse sobre identidades entitativas sino en el ejercicio de la multiplicidad y la diferencia.

El Anti-Edipo hace una demolición esquizoanalítica del parentesco, distinguiendo entre producción (momento universal del socius) y la inscripción (registro de la producción). El problema es que, en este aspecto, Deleuze es, para Viveiros, antropocéntrico. Sigue pensando en términos de pasaje de Naturaleza a la Cultura. Por eso Viveiros dice que es incompleta. Con todo, Viveiros de Castro se sirve del análisis deleuziano del mito para acogerse al concepto de intensidad. El mito es en Deleuze el momento de la intensidad y Viveiros lo relaciona con la doble filiación que presenta el estructuralismo (siguiendo a Levi-Strauss). De esta manera evidencia las raíces amerindias del estructuralismo. Esta doble filiación le sirve para detectar el momento intensivo en la filiación del mito. Siguiendo el capítulo 3 del Anti-Edipo, Viveiros de Castro descubre que en el mito existe una "filiacción precosmológica intensa, germinal, disyuntiva, nocturna y ambigua" (2010a, p. 125), determinando así las "condiciones intesivas" (2010a, p. 128) del sistema. Lo que es intenso y primordial son los linajes filiativos ambiguos, involucionados, implicados, mientras que siguiendo a Deleuze, en el orden de lo extensivo, la filiación recibe un carácter a posteriori, administrativo y jerárquico. Por ello, "el plano intensivo del mito está poblado por filiaciones preincestuosas que ignoran la alianza. El mito es intensivo porque es (pre)incestuoso, y viceversa: la alianza es "realmente" el principio de la sociedad y el fin del mito" (Viveiros 2010a, p. 129).

Pero donde nos lleva Viveiros con esta doble filiación del pensamiento amerindio es a la falta de distinción que proporciona el mito respecto a la otredad. Por ello, continua Viveiros:

\footnotetext{
Sería preciso agregar aquí que, si ese orden intensivo no conoce distinción de persona ni de sexo, tampoco conoce distinción de especie, y especialmente distinción entre humanos y no-humanos: en el mito, todos los actuantes ocupan un campo interaccional único, a la vez ontológicamente heterogéneo sociológicamente continuo (donde toda cosa es humana, lo humano es otra cosa). (Viveiros 2010a, p. 130)
}

Si en Metafísicas Caníbales Viveiros nos presenta el diálogo teórico del orden mítico con el análisis deleuziano, en The Crystal Forest (2007) Viveiros hace un ejercicio emírico sobre el 
mito, partiendo del chamanismo amerindio. Se trata de un texto fundamental para entender el cromatismo de la multiplicidad en la teoría perspectivista. El mito es allí la narrativa sobre la indiscernibilidad precosmológica entre especies, el relato no representativo sobre la virtualidad del mundo de los espíritus. Viveiros de Castro sigue de cerca el testimonio del chamán yanomami Davi Kopenawa. Con el término Xapiripë Kopenawa (2004) se refiere a los espíritus que danzan para los chamanes desde tiempos primordiales y que siguen danzando hoy en día. Se trata de espíritus luminosos, que se presentan como destellos, como imágenes de los ancestros, reflejos de una multiplicidad aún insatisfecha. Son los espíritus cuyo estatuto ontológico no podría definirse en los términos entitativos de Quine, sino, más bien, reconociendo la diferencia como afirmación ontológica deleuziana. Es en el mito donde estos espíritus son advertidos en todo su potencial transformativo. Viveiros de Castro indica que en el mito, relato del no tiempo, las potencialidades presentan lo semejante y lo diferente antes de su decantación:

I am referring to the fact that the actants of origin myths are defined by their intrinsic capacity to be something else; in this sense, each mythic being differs infinitely from itself, given that it is posited by mythic discourse only to be substituted, that is, transformed. It is this self-difference which defines a spirit, and which makes all mythic beings into spirits too. The supposed indifferentiation between mythic subjects is a function of their radical irreducibility to fixed essences or identities, whether these are generic, specific, or individual (recalling here the detotalised or 'disorganised' bodies that thrive in myths). In sum, myth posits an ontological regime commanded by a fluent intensive difference which incides on each point of a heterogenic continuum, where transformation is anterior to form, relation is superior to terms, and interval is interior to being (Viveiros de Castro, 2007, p. 18)

Así, el reto del perspectivismo es, renunciando al régimen de representación, construir un la alianza como síntesis disyuntiva, es decir, retomar el discurso estructuralista del parentesco (según vimos con sus raíces amerindias) y las aportaciones de Wagner y Strathern para indicar que el centro del pensamiento amerindio es la transformación anticipada y la afinidad potencial: lo semejante es la oportunidad para crear algo diferente; la similitud es el medio de la diferencia (Levi-Strauss, 2008).

\section{Reflexiones finales}

Viveiros de Castro presenta una propuesta que ha encontrado cabida en el giro ontológico de la antropología desde el momento en que asume el paradigma post-estructuralista (y postmetafísico, dada la crítica a cualquier sistema metafísico entitativo) y busca las raíces amerindias del estructuralismo. De esta manera, la descolonización epistémica de la antropología se convierte en una tarea permanente, aunque posiblemente incompleta, dado el carácter académico de la misma como se ha señalado desde la antropología crítica (Martins y Feronato, 2017). El perspectivismo de Viveiros tiene una estrecha relación con el cuestionamiento de varias categorías de la antropología como son el parentesco, la subjetividad, la consideración de los nohumanos, la constitución de la mismidad y la alteridad, etc. Así podemos ver su influencia en la investigación sobre la conversión cristiana amazónica de la mano de Aparecida Vilaça (2016), el grafismo amerindio de Els Lagrou (2007), los procesos de metamorfosis chamánica, etc.

Deleuze aparece como el gran interlocutor de Viveiros de Castro. Dar cuenta de la multiplicidad es la preocupación inicial del antropólogo brasileño y el reconocimiento ontológico de la diferencia en Deleuze es el clivaje oportuno para proyectar el perspectivismo 
(Viveiros de Castro, 2010b). Así, el proyecto de una antropología cromática resplandece entre las sobras de la destrucción de la metafísica, procurando hacer presente los mundos negados, decididamente incompletos de las sociedades humanas.

Como tarea pendiente e ineludible aparece el cuestionamiento del llamamiento unívoco de Viveiros de Castro. Es decir, si aceptamos la multiplicidad inherente como premisa básica del perspectivismo no podríamos negar esa misma multiplicidad metodológica en relación con el pensamiento amerindio. Por ello, por un lado, el diálogo postergado con la fenomenología aparece como cometido inevitable. No sólo con Ingold, sino con la fenomenología de lo dado (Marion 2008), que aparece como superación de esa metafísica occidental tan denostada por Viveiros de Castro. Además, propuestas como la de Michael Taussig (1993) pueden ayudarnos a entender la constitución de la identidad en términos no entitativos sin recurrir consolidar un perspectivismo sistemático. Por otra parte, aceptar la multiplicidad deleuziana implica preguntarnos por la unicidad del pensamiento amerindio. La investigación arqueológica y ecológica arroja luz sobre los diferentes modelos de etnogénesis en la región amazónica cuestionando el alcance de las dinámicas predatorias de los grupos Tupi (Hornborg 2005; Heckenberger y Góes 2009). Es decir, la relación con el otro en la región amazónica podría entenderse en términos no canibalísticos. Los grupos Arawak, por ejemplo, estaban más preocupados en establecer alianzas que en canibalizar al otro.

Con todo, el cromatismo de Deleuze brilla en la antropología contemporánea gracias a la obra de Viveiros de Castro. La renuncia al régimen de representación, la afirmación ontológica de la diferencia y la producción de un perspectivismo constitutivo nos ofrecen pistas y claves para pensar las sociedades humanas más allá de tipologías ordenantes y taxonomías conceptuales.

\section{Referencias}

ANDRADE, O. A Utopia Antropofágica. São Paulo: Globo, 1995.

ÅRHEM, K. Ecosofía Makuna. In: CORREA, F. (Ed.). La selva humanizada: ecología alternativa en el trópico húmedo colombiano. Bogotá: Instituto Colombiano de Antropología/ Fondo FEN Colombia/ Fondo Editorial CEREC, 1993.

BISET, E. Ontología de la diferencia. In: BISET, E.; FARRÁN, R. Ontologías políticas. Buenos Aires: Imago Mundi, 2011.

BOUHABEN, M. A. Canibalizar a Deleuze. La digestión de los conceptos postestructuralistas en el pensamiento de Viveiros de Castro. RELASO, v. 7, n. 2, 2017, p. 91-102. DOI: https://doi.org/10.17979/relaso.2017.7.2.3070.

CLASTRES, P. Arqueología da violência. São Paulo: Cosac Naify, 2004.

DELEUZE, G.; GUATTARI, F. El Anti-Edipo: capitalismo y esquizofrenia. Barcelona: Paidós, 1985. Qu'est-ce que la philosophie? Paris: Minuit, 1991.

DELEUZE, G. Diferencia y repetición. Buenos Aires: Amorrortu, 2002. . Empirismo y subjetividad. Barcelona: Gedisa, 1996. 
DERRIDA, J. La différance. In: Márgenes de la filosofía. Madrid: Cátedra, 1989.

GARCÍA, J.; VINOLO, S. El resplandor de la selva invisible: hacia una fenomenología de las significaciones invistas. Antípoda. Revista de Antropología y Arqueología, n. 33, 2018, p. 125144. https://doi.org/10.7440/antipoda33.2018.07.

GODDARD, J. F. Metafísicas caníbales. Viveiros de Castro, Deleuze y Spinoza. Traducción de Carolina Villada Castro. Cuadernos de Filosofía Latinoamericana, v. 37, n. 114, 2016, p. 205-211. Original: "Métaphysiques cannibales. Viveiros de Castro, Deleuze et Spinoza". Document de travail ERRAPHIS. Disponível em: $\underline{\text { http://erraphis.univ- }}$ tlse2.fr/servlet/com.univ.collaboratif.utils.\%20LectureFichiergw?ID_FICHIER=1317125385801

HORNBORG, A. Ethnogenesis, regional integration, and ecology in prehistoric Amazonia: toward a system perspective 1. Current Anthropology, v. 46, v. 4, 2005, p. 589-620.

Heckenberger, M. y Góes Neves, E. 2009. “Amazonia: archaeology”. Annu. Rev. Anthropol. 38: 251-266.

INGOLD, T. On human correspondence. $J$ R Anthropol Inst, n. 23, 2017, p. 9-27. Doi:10.1111/1467-9655.12541

The perception of environment. Essays of livelihood, dweling and skill. Londres, Nueva York: Routledge, 2000.

KOPENAWA, D. Y. Xapiripë. In: ALBER, T. B.; KOPENAWA, D. Yanomami, o Espírito da Floresta. Rio de Janeiro: Centro Cultural Banco do Brasil / Fondation Cartier, 2004.

LAGROU, E. A fluidez da forma: arte, alteridade e agência em una sociedade amazônica (Kaxinawa, Acre). Río de Janeiro: Topbooks, 2007.

LATOUR, B. Enquete sur les modes d 'existence. Paris: La Découverte, 2012. p. 21-22.

Perspectivism: Type or Bomb? Anthropology Today, guest editorial, v. 25, n. 2, 2009, . Nous n'avons jamais été modemes. París: La Découverte, 1991.

LEVI- STRAUSS, C. Ouvres. Paris: Gallimard, 2008.

Postface. L'Homme, 154-155, 2000, p. 713-720.

MARION, J-L. Siendo dado, ensayo para una fenomenología de la donación. Madrid: Síntesis, 2008.

MARRIOT, M. Hindu Transactions: Diversity without Dualism. In: KAPFERER, B. (Ed.). Transactions and Meaning: Directions in the Anthropology of Exchange and Symbolic Behavior. Philadelphia: ISHI Publications, 1976.

MARTINS, A.; FERONATO, J. Política e (in)disciplina. ¿Por qué Viveiros de Castro nos conmueve? Tabula Rasa, n. 27, 2017, p. 483-497. https://doi.org/10.25058/20112742.461. 
STRATHERN, M. "Future kinship and the study of culture". In: Reproducing the future: Anthropology, kinsship, and the new reproductive technology. Nueva York: Routledge, 1992a. p. 43-63.

STRATHERN, M. Parts and wholes: Refiguring relationships in a post plural world. In: Reproducing the future: Anthropology, kinship, and the new reproductivo tecfmologies. Nueva York: Routledge, 1992b. p. 90-116.

The Gender of the Gift: Problems with Women and Problems with Society in Melanesia. Berkeley: University of California Press, 1988.

TAYLOR, A. C. Voir comme un autre: gurations amazoniennes de l'âime et des corps. In: DESCOLA, P. (Ed.) La fabrique des images. Visions du monde et formes de la representácion. París: Musée du Quai Branly, 2010. p. 41-50.

TAYLOR, A. C. Don Quichotte en Amérique. In: IZARD, M. (Eel.). Lévi-Stratuss. París: fid. de L'Herne, 2004. p. 92-98.

TAUSSIG, M. Mimesis and alterity. London: Routledge, 1993.

VILAÇA, A. Praying and Preying. Christianity in indigenous amazonia. Oakland: University of California Press, 2016.

VIVEIROS DE CASTRO, E. La Mirada del jaguar. Introducción al perspectivismo amerindio. Buenos Aires: Tinta y Limón, 2013.

The Inconstancy of the Indian Soul: The Encounter of Catholics and Cannibals in Sixteenth-century Brazil, Cambridge: Prickly Paradigm Press, 2011.

. Metafísicas caníbales. Buenos Aires: Katz, 2010a.

Intensive Filiation and Demonic Alliance. In: JENSEN, C. B.; RÖDJE, K. (Eds.). Deleuzian Intersections. Science, Technology, Anthropology. New York, Oxford: Berghahn Books, 2010b.

. The Crystal Forest: Notes on the Ontology of Amazonian Spirits. Inner Asia 9 (2), 2007, p. 153 - 172. Doi: 10.1163/146481707793646575

. From the enemy's point of view. Chicago: University Chicago Press, 1992.

WAGNER, R. The invention of culture. Chicago: University Chicago Press, 1981. 